\section{Baixo peso ao nascer no Brasil de acordo com as informações sobre nascidos vivos do Ministério da Saúde, 2005}

\author{
Low birth weight in Brazil according to live birth \\ data from the Ministry of Health, 2005
}

\footnotetext{
${ }^{1}$ Escola Nacional de Saúde Pública Sergio Arouca, Fundação Oswaldo Cruz Rio de Janeiro, Brasil. 2 Instituto de Comunicação e Informação Científica e Tecnológica em Saúde, Fundação Oswaldo Cruz, Rio de Janeiro, Brasil. 3 Faculdade de Medicina, Universidade de São Paulo, São Paulo, Brasil.

Correspondência C. L. T. Andrade Departamento de Administração e Planejamento em Saúde Escola Nacional de Saúde Pública Sergio Arouca Fundação Oswaldo Cruz. Rua Leopoldo Bulhões 1480, Rio de Janeiro, $R J$ 21041-210, Brasil. carlamv@ensp.fiocruz.br
}

\begin{abstract}
Although the Brazilian Information System on Live Births (SINASC) has expanded its coverage and improved its data quality since the system was implemented, the live birth count in Brazil is still not complete. The current study analyzes inequalities in low birth weight in Brazil in 2005, based on geographic factors, municipality of residence, maternal schooling, and prenatal care. An analysis of all live births showed a low birth weight paradox, namely high percentages in areas with the highest socioeconomic development. The main explanation for these paradoxical findings involves shorter neonatal survival and inadequate recording of premature births in poorer municipalities. Considering at-term live births from non-multiple gestations, inequalities were found in maternal schooling. Comprehensive, quality prenatal care could have a greater impact by reducing negative outcomes of gestation and decreasing the socioeconomic inequalities of perinatal health in Brazil.
\end{abstract}

Live Birth; Low Birth Weight Infant; Information Systems
Carla Lourenço Tavares de Andrade 1

Celia Landmann Szwarcwald 2

Euclides Ayres de Castilho ${ }^{3}$

\section{Introdução}

O Sistema de Informação sobre Nascidos Vivos (SINASC), implantado no ano de 1990 pelo Ministério da Saúde, tem como base a Declaração de Nascido Vivo, documento cuja emissão é obrigatória no serviço de saúde onde ocorreu o parto. Diferentemente do sistema de nascimentos do Registro Civil, cujo objetivo principal é a contagem do número de registros de nascimentos, o SINASC tem como propósito caracterizar as condições de nascimento, de acordo com alguns fatores como peso ao nascer, duração da gestação, tipo de parto, idade da mãe e paridade que, reconhecidamente, influenciam o estado de saúde da criança.

Atualmente, o SINASC está implantado em todos os estados brasileiros, e as informações são divulgadas pela página da Internet do Departamento de Informática do SUS (DATASUS; http://www.datasus.gov.br), estando disponíveis por município de 1994 a 2005. Embora seja perceptível que a cobertura do SINASC esteja crescendo e que a qualidade da informação venha melhorando desde a sua implantação, sabe-se que a cobertura do sistema ainda não é completa, tendo sido estimada em $89 \%$ no ano de 2004 (DATASUS. http://tabnet.datasus.gov.br/cgi/ idb2006/f10.htm, acessado em 16/Ago/2007). A análise dos dados de nascidos vivos mostra ainda deficiências na cobertura do SINASC em alguns municípios do país, embora de menor magni- 
tude que as apresentadas pelo Sistema de Informações sobre Mortalidade (SIM) do Ministério da Saúde 1 .

Um expressivo número de estudos, tanto da literatura nacional como na estrangeira, tem mostrado que filhos de mães socialmente desfavorecidas têm um maior risco de baixo peso ao nascer 2,3,4. A análise dos dados do SINASC permite verificar a ocorrência do baixo peso ao nascer no Brasil, bem como estabelecer as desigualdades socioespaciais no território nacional.

O peso do bebê ao nascimento é fortemente associado ao risco de morrer no primeiro ano de vida 5 e em grau menor, com problemas de desenvolvimento na infância, além da maior probabilidade de várias doenças na vida adulta 6 . Controlando-se ou não por idade gestacional, a proporção de baixo peso ao nascer é também o indicador mais comumente utilizado para avaliar os cuidados no pré-natal 7.

Paradoxalmente, apesar da associação da ocorrência de baixo peso ao nascer com o menor nível sócio-econômico, as análises em subgrupos populacionais específicos têm mostrado correlações em sentido contrário. Estudos desenvolvidos nos Estados Unidos mostraram que as proporções de baixo peso ao nascer são menores em populações de menor nível sócio-econômico, como entre os mexicanos residentes naquele país 3,8,9. De maneira similar, estudo no Brasil, comparando dados de nascidos vivos notificados em São Luís (Maranhão) e Ribeirão Preto (São Paulo) 10 revelou que as regiões mais desenvolvidas do Brasil detêm maiores taxas de baixo peso ao nascer do que as regiões menos desenvolvidas.

No presente estudo, analisam-se as desigualdades da proporção de baixo peso ao nascer, no Brasil, em 2005. As desigualdades são examinadas à luz de alguns recortes como o geográfico, tamanho da população do município, e escolaridade da mãe. Examina-se, ainda, a influência da atenção pré-natal em amenizar as desigualdades.

\section{Material e métodos}

As informações usadas neste estudo foram provenientes do SINASC para todos os municípios brasileiros em 2005, último ano com informações disponíveis. A análise foi realizada por Grande Região e porte populacional (municípios com menos de 50 mil habitantes e municípios com 50 mil habitantes ou mais).

As informações da mãe e da criança foram analisadas utilizando-se as seguintes variáveis: peso ao nascer (eliminando-se da análise nascidos vivos com peso ao nascer $<500 \mathrm{~g}$ ou peso ao nascer $>6.000 \mathrm{~g}$ ), idade gestacional ( $<32$ semanas, 32 a 36 semanas, $\geq 37$ semanas), tipo de gravidez (não-múltipla, múltipla), número de consultas de pré-natal (nenhuma consulta, 1 a 6 consultas, 7 ou mais consultas), tipo de parto (vaginal, cesáreo) e grau de escolaridade da mãe (Ensino Fundamental incompleto: $<8$ anos de estudo; Ensino Fundamental completo: $\geq 8$ anos de estudo). A forma de categorização das variáveis obedeceu à disponibilidade das informações do SINASC.

Com base nessas variáveis, foram construídos os indicadores: percentual de baixo peso ao nascer (peso $<2.500 \mathrm{~g}$ ), percentual de prematuridade ( $<37$ semanas de gestação), percentual de mães sem consulta de pré-natal, percentual de mães com 7 ou mais consultas de pré-natal, percentual de partos cesáreos, percentual de mães com Ensino Fundamental incompleto.

\section{Resultados}

Na Tabela 1, os indicadores são apresentados segundo região e categoria populacional. Analisando-se a variável relativa ao grau de escolaridade das mães, percebem-se as grandes desigualdades sócio-espaciais: os percentuais mais elevados de baixa escolaridade encontram-se nas regiões Norte e Nordeste, e dentro das regiões, nos municípios com menos de 50 mil habitantes. Chama a atenção que na Região Nordeste $61,8 \%$ das mães têm Ensino Fundamental incompleto e, entre as que residem nos municípios de menor porte, o percentual alcança $72 \%$.

Com relação à assistência pré-natal, os indicadores obedecem a um gradiente sociogeográfico similar. No que se refere ao percentual de mães com 7 ou mais consultas, os municípios com 50 mil habitantes ou mais de todas as regiões são os que apresentam os maiores valores. Esse indicador varia de 33,8\% na Região Norte a $69,9 \%$ na Região Sudeste. Já o percentual de mães sem atendimento pré-natal chega a atingir $8 \%$ entre os municípios com menos de 50 mil habitantes da Região Norte. É importante enfatizar, porém, que nas regiões mais desenvolvidas do país as proporções de nenhuma consulta são ligeiramente menores entre as mães residentes nos municípios de pequeno tamanho populacional.

Os dados da Tabela 1 mostram uma inversão do gradiente sócio-espacial para a ocorrência de baixo peso ao nascer: os municípios com população de 50 mil habitantes ou mais apresentam, sem exceção, percentuais maiores do que os municípios com menos de 50 mil habitantes, para todas as macrorregiões geográficas. Merece 
Indicadores selecionados construídos com base em informações do Sistema de Informação sobre Nascidos Vivos (SINASC) segundo Grande Região e categoria populacional. Brasil, 2005.

\begin{tabular}{|c|c|c|c|c|c|c|}
\hline $\begin{array}{l}\text { Região/Categoria } \\
\text { populacional } \\
\text { (habitantes) }\end{array}$ & $\begin{array}{c}\text { Mães com } \\
\text { Ensino Fundamental } \\
\text { incompleto (\%) }\end{array}$ & $\begin{array}{c}\text { Mães sem } \\
\text { consulta } \\
\text { pré-natal (\%) }\end{array}$ & $\begin{array}{c}\text { Mães com } \\
7 \text { ou mais consultas } \\
\text { de pré-natal (\%) }\end{array}$ & $\begin{array}{c}\text { Baixo peso } \\
\text { ao nascer } \\
\text { (\%) }\end{array}$ & $\begin{array}{l}\text { Prematuridade } \\
\text { (\%) }\end{array}$ & $\begin{array}{c}\text { Parto } \\
\text { cesáreo } \\
\text { (\%) }\end{array}$ \\
\hline \multicolumn{7}{|l|}{ Norte } \\
\hline$<50.000$ & 70,6 & 8,0 & 22,0 & 5,9 & 4,8 & 25,2 \\
\hline$\geq 50.000$ & 51,7 & 5,0 & 33,8 & 7,3 & 5,4 & 37,2 \\
\hline Total & 59,2 & 6,2 & 29,1 & 6,8 & 5,2 & 32,4 \\
\hline \multicolumn{7}{|l|}{ Nordeste } \\
\hline$<50.000$ & 72,3 & 4,0 & 30,4 & 6,5 & 5,1 & 24,7 \\
\hline$\geq 50.000$ & 51,6 & 3,4 & 41,5 & 8,3 & 6,2 & 39,3 \\
\hline Total & 61,8 & 3,7 & 36,0 & 7,4 & 5,7 & 32,1 \\
\hline \multicolumn{7}{|l|}{ Sudeste } \\
\hline$<50.000$ & 51,3 & 1,2 & 60,8 & 8,2 & 6,3 & 48,7 \\
\hline$\geq 50.000$ & 34,4 & 1,5 & 69,9 & 9,2 & 7,8 & 52,6 \\
\hline Total & 38,1 & 1,4 & 67,9 & 9,0 & 7,5 & 51,7 \\
\hline \multicolumn{7}{|l|}{ Sul } \\
\hline$<50.000$ & 51,8 & 0,8 & 66,8 & 7,8 & 6,4 & 49,1 \\
\hline$\geq 50.000$ & 37,6 & 1,5 & 69,3 & 8,9 & 8,0 & 50,3 \\
\hline Total & 43,2 & 1,2 & 68,3 & 8,4 & 7,3 & 49,8 \\
\hline \multicolumn{7}{|l|}{ Centro-oeste } \\
\hline$<50.000$ & 53,5 & 1,4 & 58,7 & 6,4 & 5,3 & 47,3 \\
\hline$\geq 50.000$ & 38,4 & 1,9 & 60,4 & 8,1 & 7,4 & 50,5 \\
\hline Total & 43,7 & 1,7 & 59,8 & 7,5 & 6,7 & 49,4 \\
\hline \multicolumn{7}{|l|}{ Brasil } \\
\hline$<50.000$ & 62,6 & 3,1 & 44,2 & 7,1 & 5,5 & 35,7 \\
\hline$\geq 50.000$ & 40,8 & 2,3 & 58,8 & 8,7 & 7,2 & 47,5 \\
\hline Total & 48,5 & 2,6 & 53,6 & 8,1 & 6,6 & 43,3 \\
\hline
\end{tabular}

destacar que as regiões Sudeste e Sul são as que possuem percentuais mais elevados de baixo peso ao nascer, atingindo valores de $9 \%$ entre os municípios de maior tamanho populacional.

No que diz respeito à análise dos dados de idade gestacional por região e categoria populacional, a freqüência relativa de prematuridade apresenta comportamento semelhante ao percentual de baixo peso ao nascer (Tabela 1). $\mathrm{O}$ percentual mais elevado de nascidos vivos prematuros é observado nos municípios com 50 mil habitantes ou mais da Região Sul (8\%).

$\mathrm{O}$ indicador referente ao tipo de parto, apresentado também na Tabela 1, revela que o percentual de parto cesáreo é maior nos municípios com 50 mil habitantes ou mais em todas as regiões, variando de $37,2 \%$ na Região Norte a 52,6\% na Sudeste.

O percentual de baixo peso ao nascer por idade gestacional segundo categoria populacional e região é apresentado na Tabela 2. É interessante notar que entre os nascidos vivos com menos de 32 semanas de gestação os percentuais de baixo peso ao nascer são, invariavelmente, maiores do que $95 \%$ e entre os nascidos a termo, menores do que 5\%. Contrariamente, há uma grande variação nos percentuais de baixo peso ao nascer entre os nascidos vivos com idade gestacional entre 32 e 36 semanas, de 34,7\% na Região Norte, nos municípios pequenos, a 59,2\% na Nordeste, nos municípios grandes. Analisando-se o indicador por categoria populacional, chamam a atenção também as diferenças intra-regionais na proporção de baixo peso ao nascer na categoria de idade gestacional 32-36 semanas, em que são observados para todas as macrorregiões geográficas percentuais mais elevados nos municípios com 50 mil habitantes ou mais.

Na Tabela 3, apresenta-se o percentual de baixo peso ao nascer por grau de escolaridade da mãe, por categoria populacional e região, entre os nascidos vivos com 37 ou mais semanas de gesta- 


\begin{tabular}{|c|c|c|c|}
\hline \multicolumn{4}{|c|}{ Percentual de baixo peso ao nascer por idade gestacional segundo Grande Região e categoria populacional. Brasil, 2005.} \\
\hline \multirow{2}{*}{$\begin{array}{l}\text { Região/ldade gestacional } \\
\text { (semanas) }\end{array}$} & \multicolumn{2}{|c|}{ Baixo peso ao nascer (\%) } & \multirow[t]{2}{*}{ Total } \\
\hline & $<50.000$ habitantes & $\geq 50.000$ habitantes & \\
\hline \multicolumn{4}{|l|}{ Norte } \\
\hline$<32$ & 97,8 & 96,1 & 96,7 \\
\hline $32-36$ & 34,7 & 55,1 & 47,4 \\
\hline 37 ou mais & 4,1 & 4,2 & 4,2 \\
\hline Total & 5,9 & 7,3 & 6,8 \\
\hline \multicolumn{4}{|l|}{ Nordeste } \\
\hline$<32$ & 97,9 & 97,8 & 97,9 \\
\hline $32-36$ & 45,6 & 59,2 & 53,2 \\
\hline 37 ou mais & 4,0 & 4,4 & 4,2 \\
\hline Total & 6,5 & 8,2 & 7,3 \\
\hline \multicolumn{4}{|l|}{ Sudeste } \\
\hline$<32$ & 98,0 & 98,0 & 98,0 \\
\hline $32-36$ & 56,7 & 59,0 & 58,6 \\
\hline 37 ou mais & 4,5 & 4,4 & 4,4 \\
\hline Total & 8,2 & 9,1 & 8,9 \\
\hline \multicolumn{4}{|l|}{ Sul } \\
\hline$<32$ & 98,8 & 98,7 & 98,7 \\
\hline $32-36$ & 56,0 & 59,0 & 58,0 \\
\hline 37 ou mais & 4,1 & 4,0 & 4,0 \\
\hline Total & 7,8 & 8,8 & 8,4 \\
\hline \multicolumn{4}{|l|}{ Centro-oeste } \\
\hline$<32$ & 98,4 & 98,0 & 98,1 \\
\hline $32-36$ & 51,5 & 51,7 & 51,7 \\
\hline 37 ou mais & 3,4 & 4,0 & 3,8 \\
\hline Total & 6,3 & 8,0 & 7,4 \\
\hline \multicolumn{4}{|l|}{ Brasil } \\
\hline$<32$ & 98,1 & 97,9 & 98,0 \\
\hline $32-36$ & 49,7 & 58,2 & 55,6 \\
\hline 37 ou mais & 4,1 & 4,3 & 4,2 \\
\hline Total & 7,0 & 8,6 & 8,0 \\
\hline
\end{tabular}

ção e gravidez não-múltipla. São constatadas as desigualdades do baixo peso ao nascer por grau de instrução da mãe, com percentuais de baixo peso sempre menores entre as mães de maior grau de escolaridade, qualquer que seja o estrato composto por região e categoria populacional.

Analisando-se o percentual de baixo peso ao nascer por tipo de parto segundo categoria populacional e Grande Região, entre nascidos vivos com 37 ou mais semanas de gestação e gravidez não-múltipla, observa-se que o percentual de baixo peso ao nascer é sempre menor entre as mães que realizaram partos por via cesárea do que entre aquelas com parto vaginal (Tabela 4).

$\mathrm{Na}$ Tabela 5, pode-se analisar o percentual de baixo peso ao nascer por atendimento prénatal segundo região de residência e grau de escolaridade da mãe. A tendência de decréscimo da proporção de baixo peso ao nascer com o aumento do número de consultas é constatada em todas as regiões e nas duas categorias de nível de instrução. Na Região Sul, entre as mães sem atendimento pré-natal e com Ensino Fundamental incompleto, o percentual de baixo peso ao nascer atinge $9,2 \%$, mesmo em crianças nascidas a termo.

\section{Discussão}

A análise realizada neste estudo, sobre as informações contidas no SINASC, teve o objetivo de avaliar as desigualdades sócio-espaciais do baixo peso ao nascer no Brasil. Observou-se o 
Percentual de baixo peso ao nascer por grau de escolaridade da mãe segundo Grande Região e categoria populacional entre os nascidos vivos de 37 ou mais semanas de gestação e gravidez não-múltipla. Brasil, 2005.

\begin{tabular}{|c|c|c|c|}
\hline \multirow[t]{2}{*}{ Região/Grau de escolaridade } & \multicolumn{2}{|c|}{ Baixo peso ao nascer (\%) } & \multirow[t]{2}{*}{ Total } \\
\hline & $<50.000$ habitantes & $\geq 50.000$ habitantes & \\
\hline \multicolumn{4}{|l|}{ Norte } \\
\hline Ensino Fundamental incompleto & 3,8 & 4,2 & 4,0 \\
\hline Ensino Fundamental completo & 3,1 & 3,5 & 3,4 \\
\hline Total & 3,6 & 3,9 & 3,8 \\
\hline \multicolumn{4}{|l|}{ Nordeste } \\
\hline Ensino Fundamental incompleto & 3,7 & 4,4 & 4,0 \\
\hline Ensino Fundamental completo & 3,0 & 3,5 & 3,3 \\
\hline Total & 3,5 & 4,0 & 3,7 \\
\hline \multicolumn{4}{|l|}{ Sudeste } \\
\hline Ensino Fundamental incompleto & 4,6 & 4,7 & 4,6 \\
\hline Ensino Fundamental completo & 3,5 & 3,6 & 3,5 \\
\hline Total & 4,0 & 3,9 & 4,0 \\
\hline \multicolumn{4}{|l|}{ Sul } \\
\hline Ensino Fundamental incompleto & 4,2 & 4,3 & 4,2 \\
\hline Ensino Fundamental completo & 3,0 & 3,2 & 3,1 \\
\hline Total & 3,6 & 3,6 & 3,6 \\
\hline \multicolumn{4}{|l|}{ Centro-oeste } \\
\hline Ensino Fundamental incompleto & 3,5 & 4,0 & 3,8 \\
\hline Ensino Fundamental completo & 2,6 & 3,3 & 3,1 \\
\hline Total & 3,1 & 3,6 & 3,4 \\
\hline \multicolumn{4}{|l|}{ Brasil } \\
\hline Ensino Fundamental incompleto & 3,9 & 4,4 & 4,2 \\
\hline Ensino Fundamental completo & 3,1 & 3,5 & 3,4 \\
\hline Total & 3,6 & 3,9 & 3,8 \\
\hline
\end{tabular}

paradoxo do baixo peso ao nascer com os percentuais mais baixos de baixo peso ao nascer nas localidades menos favorecidas socialmente. Os resultados confirmam achados anteriores encontrados no Brasil, nos Estados Unidos e na Noruega 9,10,11,12. Tanto no que se refere à análise por Grande Região como por categoria populacional, foram, invariavelmente, encontrados maiores percentuais de baixo peso ao nascer nas áreas geográficas de maior desenvolvimento sócio-econômico.

Uma hipótese explicativa para o paradoxo do baixo peso ao nascer é o aumento de nascimentos pré-termo, que antes constituíam natimortos. Nas áreas menos desenvolvidas do país, devido à atenção inadequada e precariedade de acesso à tecnologia perinatal, muitos desses nascidos vivos morrem logo após o parto e, freqüentemente, não são registrados ou são classificados indevidamente como natimortos, reduzindo as proporções de prematuridade nestes locais. Conforme evidenciado em estudo realizado em oito Uni- dades da Federação 13, ainda há dificuldades em se obter indicadores para o monitoramento da mortalidade perinatal com base nas informações do Ministério da Saúde, devido, principalmente, a falhas no preenchimento das declarações de óbito, não sendo possível, muitas vezes, saber o momento da morte.

A análise por idade gestacional revelou que existem diferenças substanciais nas proporções de baixo peso ao nascer entre os nascidos vivos com 32 a 36 semanas de gestação, fornecendo evidências da subnotificação de nascidos de 32 a 36 semanas de gestação com baixo peso nas regiões Norte e Nordeste, principalmente nos municípios com menos de 50 mil habitantes. Estudo anterior realizado no Brasil, que relacionou o Sistema de Internações Hospitalares (SIH) com o SINASC 14, mostrou, igualmente, maior subregistro de nascimentos justamente nos municípios pequenos, de tal modo que quanto menor o porte populacional, tanto maior a subnotificação de nascidos vivos no SINASC. 
Percentual de baixo peso ao nascer por tipo de parto entre os nascidos vivos de 37 ou mais semanas de gestação e gravidez não-múltipla, segundo Grande Região e categoria populacional. Brasil, 2005.

\begin{tabular}{|c|c|c|c|}
\hline \multirow[t]{2}{*}{ Região/Tipo de parto } & \multicolumn{2}{|c|}{ Baixo peso ao nascer (\%) } & \multirow[t]{2}{*}{ Total } \\
\hline & $<50.000$ habitantes & $\geq 50.000$ habitantes & \\
\hline \multicolumn{4}{|l|}{ Norte } \\
\hline Vaginal & 3,8 & 4,1 & 4,0 \\
\hline Cesáreo & 3,0 & 3,4 & 3,3 \\
\hline Total & 3,6 & 3,9 & 3,8 \\
\hline \multicolumn{4}{|l|}{ Nordeste } \\
\hline Vaginal & 3,6 & 4,4 & 3,9 \\
\hline Cesáreo & 3,1 & 3,3 & 3,3 \\
\hline Total & 3,5 & 4,0 & 3,7 \\
\hline \multicolumn{4}{|l|}{ Sudeste } \\
\hline Vaginal & 4,7 & 4,3 & 4,4 \\
\hline Cesáreo & 3,3 & 3,6 & 3,6 \\
\hline Total & 4,0 & 4,0 & 4,0 \\
\hline \multicolumn{4}{|l|}{ Sul } \\
\hline Vaginal & 4,2 & 3,9 & 4,0 \\
\hline Cesáreo & 3,1 & 3,3 & 3,2 \\
\hline Total & 3,6 & 3,6 & 3,6 \\
\hline \multicolumn{4}{|l|}{ Centro-oeste } \\
\hline Vaginal & 3,6 & 4,0 & 3,8 \\
\hline Cesáreo & 2,4 & 3,2 & 3,0 \\
\hline Total & 3,1 & 3,6 & 3,4 \\
\hline \multicolumn{4}{|l|}{ Brasil } \\
\hline Vaginal & 3,9 & 4,2 & 4,1 \\
\hline Cesáreo & 3,1 & 3,5 & 3,4 \\
\hline Total & 3,6 & 3,9 & 3,8 \\
\hline
\end{tabular}

As falhas de registro de nascimentos prematuros foram também verificadas em outros países. Em estudo realizado na Suíça, os autores concluíram que um significante número de crianças com baixo peso ao nascer, cuja morte é próxima ao momento do parto, não é oficialmente registrado ${ }^{15}$. Análise da adequação dos dados provenientes dos certificados de nascimentos do Estado de New Jersey, Estados Unidos, foi realizada mediante a comparação com dados obtidos em um programa estadual de pré-natal, entre 1989 e 1992, de acordo com as características sócio-econômicas da mãe 16 . Os resultados indicaram que os piores percentuais de concordância do baixo peso ao nascer e da prematuridade ocorreram entre as mães de pior nível sócio-econômico sem proficiência na língua inglesa.

Segundo as estatísticas vitais da população norte-americana, no ano de 200417 a proporção de prematuridade aumentou de 9,4\% em 1981 para 10,6\% em 1990 e atingiu 12,5\% em 2004, refletindo a maior sobrevida dos nasci- dos vivos prematuros. Embora a mortalidade infantil nos Estados Unidos tenha mostrado relevante decréscimo nas últimas décadas, o percentual de baixo peso ao nascer tem mostrado nítido acréscimo: de 6,7\% em 1984, passou a $8,1 \%$ em 2004, acompanhando a tendência de crescimento da prematuridade. Os resultados de Barros et al. 18, que comparam indicadores de duas coortes em Pelotas (1982 e 1993), Rio Grande do Sul, indicam que o mesmo vem ocorrendo no Brasil.

Em outro trabalho realizado no Brasil, comparando os dados de Ribeiro Preto (Região Sudeste) e São Luís (Região Nordeste), foi observado o mesmo paradoxo para o baixo peso ao nascer, encontrando-se taxa significativamente maior na cidade mais rica. Fatores como determinações incorretas da idade gestacional, subregistro de nascidos vivos, bem como falhas no registro de nascidos vivos como nascidos mortos nos municípios menos desenvolvidos são considerados pelos autores como responsáveis, 
Percentual de baixo peso ao nascer por número de consultas de pré-natal e grau de escolaridade da mãe, segundo Grande Região entre os nascidos vivos de 37 ou mais semanas de gestação e gravidez não-múltipla. Brasil, 2005.

\begin{tabular}{|c|c|c|c|}
\hline \multirow{2}{*}{$\begin{array}{l}\text { Região/Número de } \\
\text { consultas de pré-natal }\end{array}$} & \multicolumn{2}{|c|}{ Baixo peso ao nascer (\%) } & \multirow[b]{2}{*}{ Total } \\
\hline & $\begin{array}{l}\text { Ensino Fundamental } \\
\text { incompleto }\end{array}$ & $\begin{array}{l}\text { Ensino Fundamental } \\
\text { completo }\end{array}$ & \\
\hline \multicolumn{4}{|l|}{ Norte } \\
\hline Nenhuma & 5,6 & 5,6 & 5,6 \\
\hline $1-6$ & 4,0 & 3,6 & 3,9 \\
\hline 7 ou mais & 3,4 & 3,1 & 3,2 \\
\hline Total & 4,0 & 3,4 & 3,8 \\
\hline \multicolumn{4}{|l|}{ Nordeste } \\
\hline Nenhuma & 6,3 & 6,0 & 6,3 \\
\hline $1-6$ & 4,2 & 3,7 & 4,0 \\
\hline 7 ou mais & 3,2 & 2,7 & 3,0 \\
\hline Total & 4,0 & 3,3 & 3,7 \\
\hline \multicolumn{4}{|l|}{ Sudeste } \\
\hline Nenhuma & 9,0 & 7,4 & 8,6 \\
\hline $1-6$ & 5,4 & 4,6 & 5,0 \\
\hline 7 ou mais & 3,9 & 3,2 & 3,4 \\
\hline Total & 4,6 & 3,5 & 4,0 \\
\hline \multicolumn{4}{|l|}{ Sul } \\
\hline Nenhuma & 9,2 & 7,8 & 8,8 \\
\hline $1-6$ & 5,0 & 4,2 & 4,6 \\
\hline 7 ou mais & 3,6 & 2,8 & 3,1 \\
\hline Total & 4,2 & 3,1 & 3,6 \\
\hline \multicolumn{4}{|l|}{ Centro-oeste } \\
\hline Nenhuma & 6,6 & 5,9 & 6,4 \\
\hline $1-6$ & 4,4 & 3,8 & 4,1 \\
\hline 7 ou mais & 3,1 & 2,7 & 2,9 \\
\hline Total & 3,8 & 3,1 & 3,4 \\
\hline \multicolumn{4}{|l|}{ Brasil } \\
\hline Nenhuma & 6,7 & 6,5 & 6,7 \\
\hline $1-6$ & 4,5 & 4,0 & 4,3 \\
\hline 7 ou mais & 3,6 & 3,0 & 3,2 \\
\hline Total & 4,2 & 3,4 & 3,8 \\
\hline
\end{tabular}

pelo menos parcialmente, pelas taxas maiores de baixo peso ao nascer nos municípios de melhor nível sócio-econômico 10.

Outras possíveis explicações para os resultados paradoxais do baixo peso ao nascer recaem no comportamento diferenciado da mulher nas áreas urbanas, tais como a freqüência maior do hábito de fumar na gestação, que é um fator, reconhecidamente, associado ao baixo peso ao nascer 6 . 0 estilo de vida, incluindo o estresse no trabalho, pode ser outro fator associado à maior ocorrência de prematuridade, e, conseqüentemente, à maior freqüência de baixo peso ao nascer 19. Dados da Pesquisa Mundial da Saúde 20, realizada no Brasil em 2003, revelaram que o percentual de mulheres que têm trabalho remunerado é significativamente maior nas cidades de grande porte populacional.

Em estudo prospectivo de coorte de nascidos vivos em 1982, 1993 e 2004, na cidade de Pelotas, mostrou-se que a prevalência de prematuridade cresceu de 6,3\% em 1982 para 16,2\% em 2004, correspondendo a uma redução de 47 gramas na média do peso ao nascer, apesar da melhoria das condições de vida materna 21 . Em vertente explicativa complementar, Barros et al. 21 consideram o papel da excessiva medicalização, incluindo indução do parto, maior proporção de partos cesáreos e ultra-sonografias imprecisas ou mal interpretadas. 
No Município do Rio de Janeiro, análise segundo a esfera administrativa do estabelecimento onde se realizou o parto (público ou privado) mostrou a influência da medicalização excessiva, além da enorme proporção de partos cesarianos ocorridos em estabelecimentos não-públicos (87\%) 22. Dados do SINASC, no mesmo município, em 2005, mostram que a proporção de bebês de baixo peso foi maior entre as mães que tiveram parto cesáreo (DATASUS. http://tabnet. datasus.gov.br/cgi/tabcgi.exe?sinasc/cnv/nvrj. def, acessado em 22/Ago/2007).

Corroborando os achados de vários estudos que mostram as disparidades do baixo peso ao nascer por nível sócio-econômico da mãe 23, os dados do SINASC, em âmbito nacional, mostram que as proporções de nascidos vivos a termo de gestação não-múltipla com peso inferior a $2.500 \mathrm{~g}$ são maiores tanto menor o grau de escolaridade da mãe, única variável representativa do nível sócio-econômico disponível no SINASC.

Um outro achado importante deste estudo foi a relevância do atendimento pré-natal no sentido de amenizar as desigualdades do baixo peso ao nascer. Assim como em trabalhos anteriores realizados em outros países da América Latina 24,25 , os dados brasileiros mostram que

\section{Resumo}

Embora seja notório que a cobertura do Sistema de Informação sobre Nascidos Vivos (SINASC) esteja crescendo e que a qualidade da informação venha melhorando, desde a sua implantação, sabe-se que a enumeração de nascidos vivos ainda não é completa no Brasil. Neste trabalho, objetiva-se analisar as desigualdades da proporção do baixo peso ao nascer no Brasil, em 2005, segundo alguns aspectos como o geográfico, o tamanho da população do município e a escolaridade da mãe. Analisou-se, igualmente, $a$ influência da atenção pré-natal. Considerando a totalidade dos nascidos vivos, evidenciou-se o paradoxo do baixo peso ao nascer, ou seja, foram encontrados percentuais mais elevados nas áreas de maior desenvolvimento sócio-econômico. Os resultados paradoxais são explicados, principalmente, pela menor sobrevida e registro inadequado dos prematuros nos municípios mais pobres. Levando-se em conta os nascidos vivos a termo de gestação não-múltipla, foram encontradas desigualdades por grau de escolaridade da mãe. Sugere-se que o atendimento pré-natal abrangente e com qualidade poderia ter um impacto maior na redução dos resultados adversos da gestação, contribuindo para a diminuição das desigualdades sócio-econômicas da saúde perinatal no Brasil.

Nascimento Vivo; Recém-Nascido de Baixo Peso; Sistemas de Informação os percentuais de baixo peso ao nascer são menores entre as mães que realizaram 7 ou mais consultas de pré-natal, número de consultas considerado adequado pelo Ministério da Saúde. Conforme discutido por Koblinsky et al. 26, o impacto do pré-natal poderia ser mais intenso, dependendo da capacidade dos serviços em identificar as gestantes de alto risco, prevenir ou tratar complicações, e referir para atenção obstétrica especializada, quando necessário.

O papel da atenção básica como elementochave para a promoção da eqüidade tem sido enfatizado, principalmente nas sociedades com grandes disparidades sociais 27 , podendo-se inferir que o atendimento pré-natal abrangente e adequado poderia ter maior impacto na redução dos resultados adversos da gestação. No caso particular deste estudo, o desempenho favorável do pré-natal indica que muito ainda pode ser feito no âmbito do setor saúde para diminuição das desigualdades do baixo peso ao nascer por grau de escolaridade no Brasil. É preciso enfatizar, ainda, a necessidade de melhora da cobertura e da qualidade das informações sobre nascidos vivos, sobretudo nas regiões menos desenvolvidas, para possibilitar a análise das informações com fidedignidade.

\section{Colaboradores}

C. L. T. Andrade foi responsável pela elaboração do texto e dos resultados. C. L. Szwarcwald participou na concepção do artigo e na elaboração do texto. E. A. Castilho participou na discussão dos resultados. 


\section{Referências}

1. Andrade CLT, Szwarcwald CL. Desigualdades sócio-espaciais da adequação das informações de nascimentos e óbitos do Ministério da Saúde, Brasil, 2000-2002. Cad Saúde Pública 2007; 23: 1207-16.

2. Gama SG, Szwarcwald CL, Leal MC, Theme-Filha $\mathrm{MM}$. The pregnancy during adolescence as a risk factor for low birth weight, Brazil. Rev Saúde Pública 2001; 35:74-80.

3. Parker JD, Schoendorf KC, Kiely JL. Associations between measures of socioeconomic status and low birth weight, small for gestational age, and premature delivery in the United States. Ann Epidemiol 1994; 4:271-8.

4. Menezes AMB, Barros FC, Victora CG, Alves C, Rocha C, Albernaz E, et al. Mortalidade perinatal em duas coortes de base populacional no Sul do Brasil: tendências e diferenças. Cad Saúde Pública 1996; 12 Suppl 1:S33-41.

5. Luginaah IN, Lee KS, Abernathy TJ, Sheehan D, Webster G. Trends and variations in perinatal mortality and low birthweight: the contribution of socio-economic factors. Can J Public Health 1999; 90:377-81.

6. Wilcox AJ. On the importance - and the unimportance - of birthweight. Int J Epidemiol 2001; 30:1233-41.

7. Fiscella K. Does prenatal care improve birth outcomes? A critical review. Obstet Gynecol 1995; 85:468-79.

8. Branum AM, Schoendorf KC. Changing patterns of low birthweight and preterm birth in the United States, 1981-98. Paediatr Perinat Epidemiol 2002; 16:8-15.

9. Buekens P, Notzon F, Kotelchuck M, Wilcox A. Why do Mexican Americans give birth to few low-birthweight infants? Am J Epidemiol 2000; 152:347-51.

10. Silva AAM, Bettiol H, Barbieri MA, Pereira MM, Brito LGO, Ribeiro VS, et al. Why are the low birthweight rates in Brazil higher in richer than in poorer municipalities? Exploring the epidemiological paradox of low birthweight. Paediatr Perinat Epidemiol 2005; 19:43-9.

11. Fuentes-Afflick E, Hessol NA, Perez-Stable EJ. Testing the epidemiologic paradox of low birthweight in Latinos. Arch Pediatr Adolesc Med 1999; 153:147-53.

12. Melve KK, Skjaerven R. Birthweight and perinatal mortality: paradoxes, social class, and sibling dependencies. Int J Epidemiol 2003; 32:625-32.

13. Almeida MF, Alencar GP, Novaes HMD, Ortiz LP. Sistemas de informação e mortalidade perinatal: conceitos e condições de uso em estudos epidemiológicos. Rev Bras Epidemiol 2006; 9:56-68.
14. Drumond EF, Machado CJ, França E. Underreporting of live births: measurement procedures using the Hospital Information System. Rev Saúde Pública 2008; 42:55-63.

15. Muller M, Drack G, Schindler C, Bucher HU. Live and stillborn very low birthweight infants in Switzerland: comparison between hospital based birth registers and the national birth register. Swiss Med Wkly 2005; 135:433-9.

16. Reichman N, Schwartz-Soicher O. Accuracy of birth certificate data by risk factors and outcomes: analysis of data from New Jersey. Am J Obstet Gynecol 2007; 197:32.e1-8.

17. Hoyert DL, Mathews TJ, Menacker F, Strobino DM, Guyer B. Annual summary of vital statistics: 2004. Pediatrics 2006; 117:168-83

18. Barros FC, Victora CG, Vaughan JP, Tomasi E, Horta BL, Cesar JA, et al. The epidemiological transition in maternal and child health in a Brazilian city, 1982-93: a comparison of two population-based cohorts. Paediatr Perinat Epidemiol 2001; 15:4-11.

19. Gabbe SG, Turner LP. Reproductive hazards of the American lifestyle: work during pregnancy. Am J Obstet Gynecol 1997; 176:826-32.

20. Leal MC, Gama SGN, Frias P, Szwarcwald CL. Healthy lifestyles and access to periodic health exams among Brazilian women. Cad Saúde Pública 2005; 21 Suppl:S78-88.

21. Barros FC, Victora CG, Barros AJD, Santos IS, Albernaz E, Matijasevich A, et al. The challenge of reducing neonatal mortality in middle-income countries: findings from three Brazilian birth cohorts in 1982, 1993, and 2004. Lancet 2005; 365:847-54.

22. Leal MC, Gama SGN, Campos MR, Cavalini LT, Garbayo LS, Brasil CLP, et al. Fatores associados à morbi-mortalidade perinatal em uma amostra de maternidades públicas e privadas do Município do Rio de Janeiro, 1999-2001. Cad Saúde Pública 2004; 20 Suppl 1:S20-33.

23. Spencer N. The effect of income inequality and macro-level social policy on infant mortality and low birthweight in developed countries - a preliminary systematic review. Child Care Health Dev 2004; 30:699-709.

24. Bortman M. Factores de riesgo de bajo peso al nacer. Rev Panam Salud Pública 1998; 3:314-21.

25. Halpern R, Barros FC, Victora CG, Tomasi E. Atenção pré-natal em Pelotas, Rio Grande do Sul, Brasil, 1993. Cad Saúde Pública 1998; 14:487-92.

26. Koblinsky MA, Tinker A, Daly P. Programming for safe motherhood: a guide to action. Health Policy Plan 1994; 9:252-66.

27. Starfield B. Improving equity in health: a research agenda. Int J Health Serv 2001; 31:545-66.

Recebido em 06/Dez/2007

Versão final reapresentada em 16/Abr/2008

Aprovado em 18/Abr/2008 